\title{
Scleral fixation of foldable acrylic intraocular lenses in aphakic post-vitrectomy eyes
}

This article was published in the following Dove Press journal:

Clinical Ophthalmology

23 December 2010

Number of times this article has been viewed

\section{Tetsuya Mutoh \\ Yukihiro Matsumoto \\ Makoto Chikuda \\ Department of Ophthalmology, Dokkyo Medical University Koshigaya Hospital, Koshigaya, Saitama, Japan}

Correspondence: Tetsuya Mutoh Department of Ophthalmology, Dokkyo Medical University, Koshigaya Hospital, Koshigaya, Saitama 343-8555, Japan Tel +8I 48965 I I I I

Fax +8I 48965 । I 27

Email mtetsuya@dokkyomed.ac.jp
Purpose: To evaluate the outcome for scleral fixation of a foldable acrylic intraocular lens (IOL) in aphakic post-pars plana vitrectomy eyes for vitreoretinal disease.

Methods: The medical records of 15 patients were reviewed. We evaluated such factors as the underlying vitreoretinal disease, preoperative expected refraction and postoperative actual refraction, best corrected visual acuity (BCVA), corneal endothelial cell density, and intraoperative and postoperative complications.

Results: The most common cause of underlying vitreoretinal disease was retinal detachment, which was found in 8 cases. The mean refractive error was -0.10 diopters (D). The mean minimum angle of resolution ( $\log$ MAR) values of BCVA were 0.27 preoperatively and 0.14 postoperatively. The mean corneal endothelial cell density was 2400 cells $/ \mathrm{mm}^{2}$ preoperatively and 2187 cells $/ \mathrm{mm}^{2}$ postoperatively. No significant differences were observed in either the $\operatorname{logMAR}$ values of BCVA or the corneal endothelial cell density before and after surgery. No intraoperative complications occurred in any of the patients. Postoperative complications occurred in a total of 7 eyes, and the most severe complications comprised 4 cases of transient ocular hypertension.

Conclusion: The results for the scleral fixation of foldable acrylic IOLs were good in aphakic post-vitrectomy eyes.

Keywords: scleral fixation, intraocular lens, aphakic post-pars plana vitrectomy eyes, severe vitreoretinal disease

\section{Introduction}

Intraocular lenses (IOLs) are universally preferred over either aphakic spectacles or contact lenses as a method of visual rehabilitation in aphakic patients. ${ }^{1}$ However, depending on the condition of the posterior capsule and ciliary zonule, the insertion of an IOL on the capsule may simply cause such problems as IOL displacement into the vitreous cavity, decentration, or tilting. ${ }^{2}$ Therefore, scleral fixation of IOLs is required in some cases. However, the scleral fixation of an IOL can also lead to many complications, such as a decrease in the corneal endothelial cell density, ${ }^{3,4}$ refractive error after surgery, ${ }^{5}$ IOL dislocation, ${ }^{3-6}$ vitreous hemorrhage, ${ }^{3-6}$ infection through the suture, ${ }^{7}$ and retinal detachment. ${ }^{3-5}$

Although the scleral fixation of an IOL in aphakic eyes after a previous pars plana vitrectomy combined with lensectomy has become more common owing to the progress made in vitrectomy surgical techniques in recent years, only a few studies ${ }^{1,8-10}$ have so far been reported.

To fill this gap in knowledge, we retrospectively evaluated the secondary scleral fixation of a foldable acrylic IOL in aphakic eyes after previous pars plana vitrectomy combined with lensectomy for vitreoretinal disease. 


\section{Patients and methods}

The medical records of 15 patients (15 eyes; 10 men, 5 women) who underwent scleral fixation of a foldable acrylic IOL in aphakic postvitrectomy eyes were reviewed between September 2006 and November 2009. Only one experienced surgeon of the Dokkyo Medical University Koshigaya Hospital, Saitama, Japan, performed all surgery. All patients had been followed for a minimum of 6 months (mean follow-up month \pm standard deviation, $13.3 \pm 6.9$ months). The mean age of the patients was $63.3 \pm 6.7$ years. All patients had clinically significant cataracts preoperatively. The secondary implantation of an IOL was planned when the vitreoretinal disease had subsided and remained stable for a minimum of 3 months ( $7.2 \pm 4.1$ months).

The axial length was measured using the IOL Master (Carl Zeiss Meditec Co Ltd), a noncontact optical coherent biometry device. The IOL power was calculated using the SRK/T (Sanders-Retzlaff-Kraff theoretical) formula. Postoperative refraction was calculated as an awareness refraction that was converted to the spherical equivalent refraction, and the postoperative refraction, postoperative best corrected visual acuity (BCVA), and corneal endothelial cell density were measured at 6 months or longer postoperatively (15.1 \pm 5.5 months).

The underlying vitreoretinal disease, preoperative expected refraction and postoperative actual refraction, preoperative and postoperative BCVA, preoperative and postoperative corneal endothelial cell density, and rate of intraoperative and postoperative complications were examined. The decimal visual acuity data were converted into logarithms of the minimum angle of resolution (logMAR) values. Transient ocular hypertension was defined as a pressure over $25 \mathrm{mmHg}$, and transient ocular hypotension was defined as that under $5 \mathrm{mmHg}$. "Transient" means a certain period of time ranging from a few days to 2 weeks at most.

\section{Surgical technique}

The surgical technique was individualized because of the complicated nature of the eyes due to a history of previous surgery. An infusion cannula utilizing a 20-gauge vitrectomy system was used to prevent eyeball collapse during surgery. The infusion cannula was placed $3.5 \mathrm{~mm}$ from the limbus in the inferotemporal quadrant before scleral fixation to clear the retropupillary space and maintain the intraocular pressure (IOP) during surgery. In 2 patients who had been administered intraocular silicone oil, the scleral fixation of the IOL was performed after the pars plana removal of the silicone oil.
A $3.5 \mathrm{~mm}$ superior scleral tunnel incision was made in all patients. The anterior chamber was filled with sodium hyaluronate $1 \%$ or sodium hyaluronate $3.0 \%$-chondroitin sulfate $4.0 \%$. An ab externo method with a 27 -gauge needle was used. After a conjunctival peritomy was performed, 2 partial thickness limbus-based triangular scleral flaps were created opposite each other at the 8 o'clock and 2 o'clock positions. The intraocular part of the suture was pulled out through the scleral incision. The free ends of the sutures were tied to the 2 haptics at the site corresponding to the maximum spread of each haptic of a foldable acrylic IOL (VA-65BB, Hoya Corp). The VA-65BB IOL has a $6.5 \mathrm{~mm}$ optic, an overall length of $13.0 \mathrm{~mm}$, and poly(methyl methacrylate) haptics with a microscopic indentation $1.3 \mathrm{~mm}$ from the tip (Figure 1). The IOL was secured by slowly pulling the external sutures to introduce the haptics into the ciliary sulcus. The needle attached to the 10-0 polypropylene suture was then passed through the partial thickness of the sclera, and the suture was then tied to itself. The loop of the knot was buried under the scleral flap.

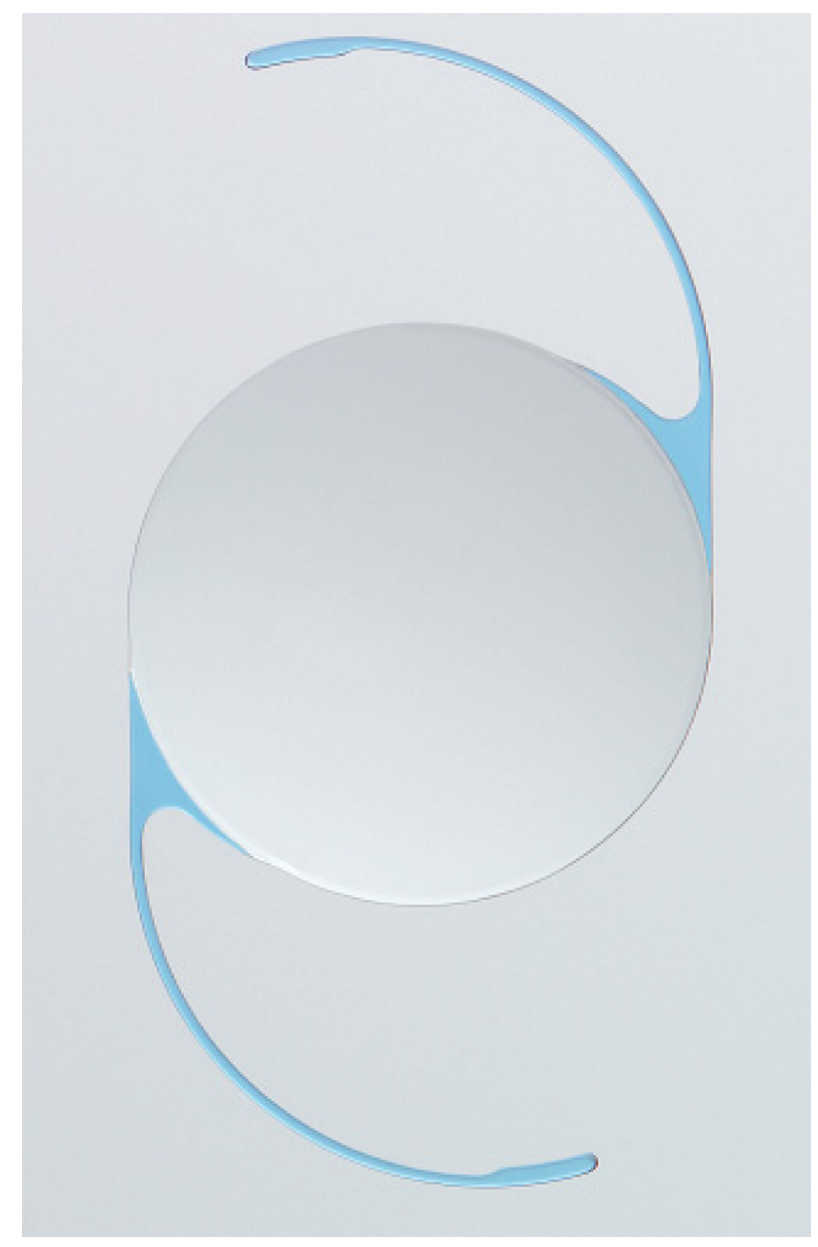

Figure I The foldable acrlylic intraocular lens has distended poly(methyl methacrylate) haptics, and each haptic has a microscopic indentation $1.3 \mathrm{~mm}$ from the tip. 
After irrigation/aspiration was done to remove any residual viscoelastic material, a balanced salt solution was injected from the corneal side port to control the IOP and observe the wound adaptation. In eyes with inadequate wound sealing, a single or continuous 10-0 nylon suture was placed at the site of the scleral tunnel incision.

\section{Statistical analysis}

The paired $t$-test was used to compare the preoperative $\log$ MAR values at BCVA, and the corneal endothelial cell density with the postoperative values.

\section{Results}

\section{Underlying vitreoretinal disease}

The underlying vitreoretinal diseases were rhegmatogenous retinal detachment ( $\mathrm{n}=8$ eyes; $53.3 \%$ ); proliferative diabetic retinopathy ( $\mathrm{n}=3$ eyes; $20.0 \%$ ); IOL displacement into the vitreous cavity $(\mathrm{n}=2 ; 13.3 \%)$; endophthalmitis after epiretinal membrane surgery $(n=1 ; 6.7 \%)$; and eye trauma with a vitreous hemorrhage $(n=1 ; 6.7 \%)$.

\section{Preoperative expected refraction} and postoperative actual refraction

Figure 2 shows a comparison of the preoperative expected refraction with the actual postoperative refraction. An average refractive error, calculated as the preoperative expected refraction from the postoperative refraction, was $-0.10 \pm 0.77$ diopters (D) (range -1.04 to $1.62 \mathrm{D}$ ).

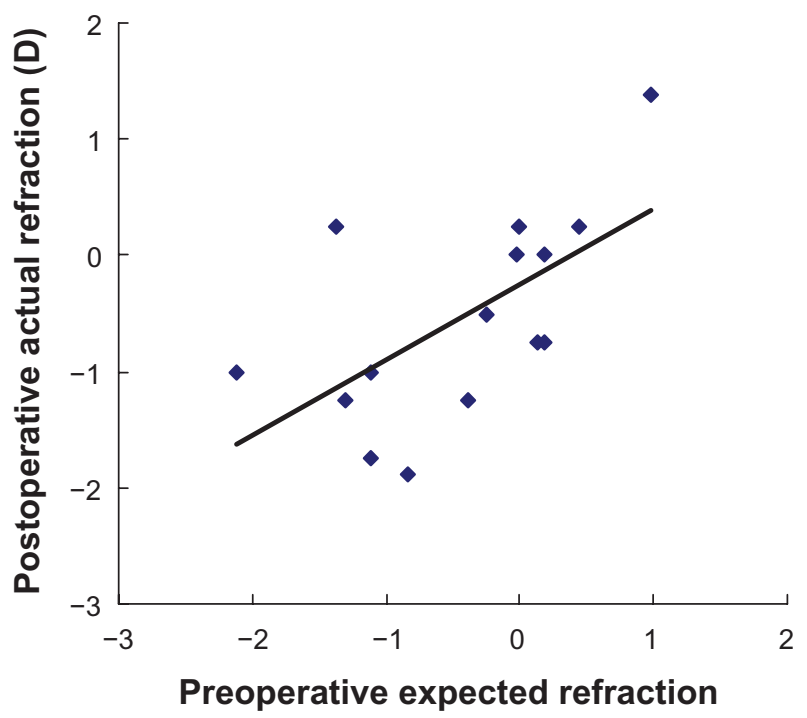

Figure 2 Preoperative expected refraction and postoperative actual refraction. The vertical axis represents the postoperative actual refraction, and the horizontal axis represents the preoperative expected refraction.

\section{Preoperative and postoperative BCVA}

The preoperative BCVAs ranged from 0.03 to 1.2 , and the postoperative best corrected decimal visual acuities ranged from 0.09 to 1.2. Figure 3 shows the preoperative and postoperative BCVA in the $\log$ MAR value. The preoperative BCVA was $0.27 \pm 0.42$ (range -0.079 to 1.522 ), and the postoperative BCVA was $0.14 \pm 0.31$ (range -0.079 to 0.398). No significant difference was seen between the eyes before and after the surgery $(P=0.05$; paired $t$-test $)$.

\section{Preoperative and postoperative corneal endothelial cell density}

Figure 4 shows the preoperative and postoperative corneal endothelial cell density. The mean preoperative corneal endothelial cell density was $2400 \pm 199$ cells $/ \mathrm{mm}^{2}$ (range 1164 to $2653 \mathrm{cells} / \mathrm{mm}^{2}$ ), and the postoperative corneal endothelial cell density was $2187 \pm 238 \mathrm{cells} / \mathrm{mm}^{2}$ (range 1031 to 2646 cells $\left./ \mathrm{mm}^{2}\right)$. No significant difference was seen between the eyes before and after surgery $(P=0.10$; paired $t$-test).

\section{Intraoperative and postoperative complications}

No intraoperative complications occurred. The postoperative complications were transient ocular hypertension $(n=4)$; transient ocular hypotension $(\mathrm{n}=1)$; IOL dislocation $(\mathrm{n}=1)$; and vitreous hemorrhage $(\mathrm{n}=1)$. Because there was some overlap in these cases, we counted the total number of eyes. Although transient ocular hypertension, transient ocular hypotension, and vitreous hemorrhage resolved without treatment during the observation course, the 2 eyes with IOL dislocation required further surgery to repair the

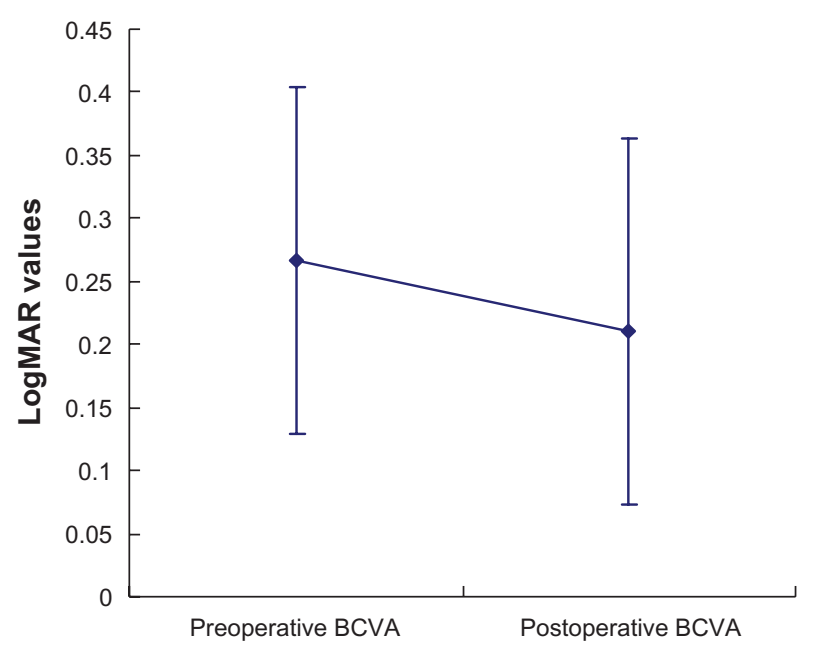

Figure 3 Preoperative and postoperative logMAR values of BCVA. The vertical axis represents the logMAR values, the horizontal axis indicates the preoperative and postoperative BCVA.

Abbreviations: BCVA, best corrected visual acuity; logMAR, mean minimum angle of resolution. 


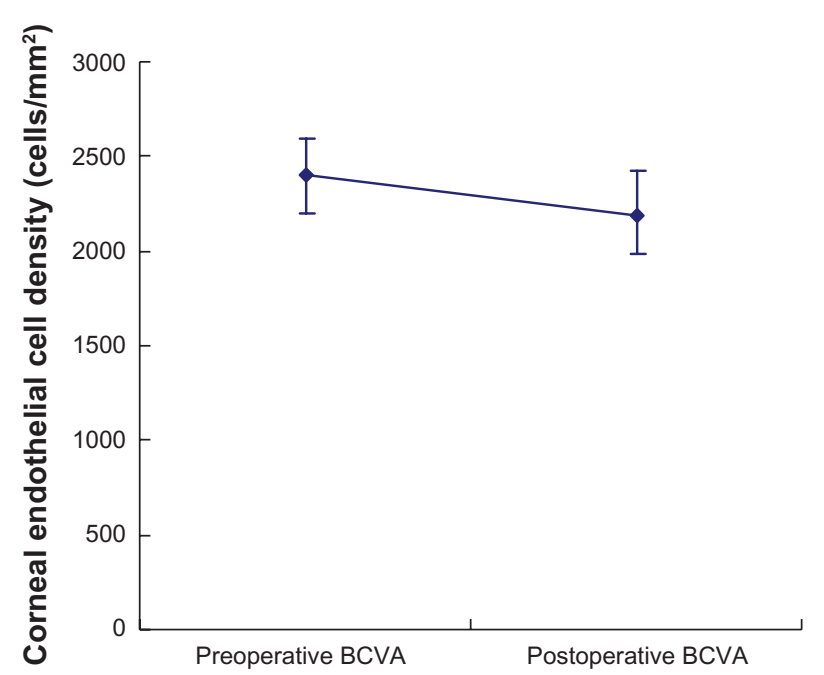

Figure 4 Preoperative and postoperative corneal endothelial cell density. The vertical axis represents the corneal endothelial cell density, while the horizontal axis indicates the preoperation and postoperation values.

dislocation. The one eye that had iris capture is still under close observation.

\section{Discussion}

The actual refractive errors reported in a previous study on the scleral fixation of IOLs in aphakic vitrectomized eyes were observed to shift approximately -0.8 (D) toward myopia in comparison with the findings observed after normal cataract surgery. ${ }^{9}$ These data are useful because used IOLs were previously limited to AQ-110NV and have been studied in many cases. ${ }^{9}$ Hotta and Hotta, in their investigation, limited the used IOLs to only one kind and focused on refractive errors. ${ }^{9}$ No controls were included in this study, thus leading to a slight shift toward myopia. However, as the refractive error was over \pm 1.50 (D) for only 2 eyes, we therefore considered these findings to be representative of good results.

In a previous study on the scleral fixation of the intraocular lenses in aphakic vitrectomized eyes reported by Ahn et al, which was similar to our present study, the postoperative BCVA significantly improved in comparison with the preoperative BCVA after 6 months. ${ }^{10}$ The postoperative BCVA was measured at 6 months or later postoperatively, and no significant difference was observed in the eyes of these patients in comparison with the preoperative BCVA described in this study.

Ohtani et al divided scleral fixation of IOL into 2 groups and compared the first group of being continuously performed after cataract surgery with the secondary group of being performed a few days after surgery. ${ }^{3}$ The postoperative corneal endothelial cell density significantly decreased at 12 months after the surgery compared with that observed before the surgery in both groups. However, no significant differences were observed between the groups. ${ }^{3}$ The postoperative corneal endothelial cell density was measured at 6 months or more postoperatively, and no significant difference in the eyes of these patients was seen in comparison with the preoperative corneal endothelial density described in this study.

Shimomura et al reported that ocular hypertension occurred in 8 of 19 eyes after scleral fixation of the IOL, but none of the eyes required glaucoma surgery. ${ }^{11}$ Krause et al reported that ocular hypertension occurred in 52 of the 119 eyes in their study after scleral fixation of the IOL, and 11 of these eyes needed subsequent glaucoma surgery. ${ }^{4}$ Fortunately, none of the eyes needed glaucoma surgery in this study. Pre-existing glaucoma tended to occur in association with the ocular hypertension, but pars plana infusion lowered the rate of ocular hypertension after surgery. ${ }^{4}$ Pars plana infusion was used in all cases in this study. No ocular hypertension after surgery occurred in any of the patients. To reduce postoperative complications, such as retinal detachment in scleral fixation of the IOL, the use of pars plana vitrectomy without severe vitreoretinal disease has lately been growing in popularity. ${ }^{11}$ Because pars plana vitrectomy itself may pose additional risks, more study is needed to establish the efficacy of the pars plana vitrectomy modality without any evidence of severe vitreoretinal disease.

Ahn et al reported the underlying vitreoretinal diseases in 21 eyes of patients who underwent scleral fixation of an IOL in aphakic vitrectomized eyes to be proliferative diabetic retinopathy in 9 eyes; proliferative vitreoretinopathy in 5 eyes; traumatic retinal detachment in 3 eyes; intraocular foreign body in 2 eyes; and uveitic retinal detachment in 2 eyes. ${ }^{10}$ Hotta and Hotta also reported the underlying vitreoretinal diseases, which included retinal detachment in 17 eyes; diabetic retinopathy in 7 eyes; and other causes in 6 eyes. ${ }^{9}$ Ahn et al reported a higher rate of proliferative diabetic retinopathy ${ }^{10}$ in comparison with this study. On the other hand, the findings reported by Hotta and $\mathrm{Hotta}^{9}$ were similar to those described in this study with regard to the underlying vitreoretinal diseases.

The VA-65BB, which is a foldable IOL that can be inserted through a $3.0 \mathrm{~mm}$ incision, was used in this study. Fass and Herman reported that large incisions were prone to demonstrate either intraoperative or postoperative hypotony, an especially dangerous situation for eyes that were essentially empty of any vitreous support. ${ }^{8}$ As P366UV and CZ70BD IOLs require large incisions, their frequency of use may decrease in the future. Fass and Herman described 
a surgical technique for aphakic postvitrectomy eyes in which a foldable acrylic IOL was used to facilitate 4-point fixation with no tilt and good centration through a 2.75 to 3.0 mm incision. ${ }^{8}$

Scleral fixation of the IOL tends to prolong the duration of surgery, thus resulting in a longer illumination of the retina by the surgical microscope. This is a risk factor for light-induced retinopathy. ${ }^{12}$ As yellow IOL protects the retina against light exposure, ${ }^{13}$ the use of a yellow IOL, either with or without pars plana vitrectomy, will therefore likely increase in the future.

There are several studies in the literature similar to this report. ${ }^{1,10}$ However, one important advantage of our study in comparison with the others is the fact that only one experienced surgeon performed all the surgery. Some differences tend to occur when different surgeons perform a large amount of surgery and thus result in reduced accuracy.

The removal of a cataract during a vitrectomy is performed to improve the surgeon's visualization. The lens capsule was removed completely during vitrectomy. It is possible to maintain the part of anterior capsule for subsequent foldable IOL implantation without sutures. However, the remaining anterior capsule has a risk of developing iridocapsular synechias, which may complicate the secondary IOL implantation and also negatively affect the occurrence of postoperative refractive errors.

However, only a few studies of this kind ${ }^{1,8-10}$ have so far been reported, and the findings presented in this study are therefore considered to be useful. We conclude that scleral fixation of foldable acrylic IOLs in aphakic postvitrectomy eyes can be safely performed because no severe problems have been found with regard to refraction, corneal endothelial cell density, or intraoperative and postoperative complications.

\section{Disclosure}

No conflicts of interest were declared in relation to this paper.

\section{References}

1. Johnston RL, Charteris DG. Pars plana vitrectomy and sutured posterior chamber lens implantation. Curr Opin Ophthalmol. 2001; 12:216-221.

2. Iino T, Ohshita M, Yamamoto T. Ab externo sulcus fixation by single unilateral suture of the intraocular lens haptics. Ganka. 2005; 47:1335-1340

3. Ohtani S, Miyata K, Ono K, et al. Outcome of sulcus fixation of the posterior chamber lens. Jpn J Clin Ophthalmol. 2000;54:531-535.

4. Krause L, Bechrakis, Heimann H, et al. Implantation of scleral fixed sutured posterior chamber lens: a retrospective analysis of 119 cases. Int Ophthalmol. 2009;29:207-212.

5. Tsuiki E, Taniguchi H, Kitaoka T, et al. Evaluation of transscleral fixation of intraocular lens. Ganka Rinsyo Ihou. 2004;98:1077-1080.

6. Ganesh A, Al-Zuhaibi S, Mitra S, et al. Visual rehabilitation by scleral fixation of posterior chamber intraocular lens in Omani children with aphakia. Ophthalmic Surg Lasers Imaging. 2009;40:354-360.

7. Tashita A, Mitamura Y, Ohtsuka K. A case of endophthalmitis 8 years after transscleral fixation of intraocular lens. Atarashii Ganka. 2004; $21: 258-260$

8. Fass ON, Herman WK. Sutured intraocular lens placement in aphakic post-vitrectomy eyes via small-incision surgery. $J$ Cataract Refract Surg. 2009;35:1492-1497.

9. Hotta K, Hotta J. Postoperative refractive error of secondary intraocular lens implantation after simultaneous vitrectomy and lensectomy. Ganka Rinsyo Ihou. 2006;100:227-230.

10. Ahn JK, Yu HG, Chung H, et al. Transscleral fixation of a foldable intraocular lens in aphakic vitrectomized eyes. J Cataract Refract Surg. 2003;29:2390-2396

11. Shimomura C, Yamada T, Noso K, et al. Cause and outcome of scleral fixation of intraocular lens. Jpn J Clin Ophthalmol. 2007; 61:1409-1412.

12. Mutoh T, Matsumoto Y, Chikuda M. Two cases of light-induced retinopathy during scleral fixation of intraocular lens. Ganka. 2009; 51:1817-1821.

13. Tanito M, Kaidzu S, Anderson RE. Protective effects of soft acrylic yellow filter against blue light-induced retinal damage in rats. Exp Eye Research. 2006;83:1493-1504.
Clinical Ophthalmology

\section{Publish your work in this journal}

Clinical Ophthalmology is an international, peer-reviewed journal covering all subspecialties within ophthalmology. Key topics include: Optometry; Visual science; Pharmacology and drug therapy in eye diseases; Basic Sciences; Primary and Secondary eye care; Patient Safety and Quality of Care Improvements. This journal is indexed on PubMed

Submit your manuscript here: http://www.dovepress.com/clinical-ophthalmology-journal

\section{Dovepress}

Central and CAS, and is the official journal of The Society of Clinical Ophthalmology (SCO). The manuscript management system is completely online and includes a very quick and fair peer-review system, which is all easy to use. Visit http://www.dovepress.com/testimonials. php to read real quotes from published authors. 